\title{
Computational Thinking in der Musikwissenschaft: Jupyter Notebook als Umgebung für Lehre und Forschung
}

UWE SEIFERT, SEBASTIAN KLASSMANN, TIMO VARELMANN UND NILS DAHMEN, KÖLN

Weltweit wird von Digitalisierung, Künstlicher Intelligenz und Roboterisierung der Gesellschaft, Wissenschaft und Kunst gesprochen. Es ist die Rede von Digital Humanities, sowie von Computational und Data Sciences. Computation ist schon jetzt eines der grundlegenden Konzepte für die Wissenschaften des 21. Jahrhunderts. ${ }^{1}$ Durch diese wissenschaftlichen Entwicklungen sowie ihre derzeitigen und zukünftigen gesellschaftlichen Auswirkungen werden grundlegende Fragen und Probleme der menschlichen Existenz sowie des menschlichen Selbstverständnisses aufgeworfen und diskutiert, die von zentraler gesellschaftlicher und kultureller Relevanz und Brisanz sind und spezifische Anforderungen an Forschung und Lehre stellen. ${ }^{2}$

Dies gilt auch für die Musikwissenschaft. So dürfte außer Frage stehen, dass sich im Zuge der Digitalisierung der Wissenschaften ihre Recherchemöglichkeiten und Quellenzugänge erweitert haben sowie "digitale Werkzeuge" nutzbar gemacht werden. ${ }^{3}$ Dies macht die anwendungsorientierte Kompetenzvermittlung in der Lehre und das Schritthalten mit digitalen Entwicklungen in der Forschung sowie im Berufsleben dringend erforderlich. Grundlegende Fragen der Musikwissenschaft und epistemologisch-methodologische Fragen des disziplinären Selbstverständnisses werden bei dieser Sichtweise der Digitalisierung als einer reinen Auffassung der Notwendigkeit eines erweiterten Werkzeuggebrauchs allerdings nicht berührt. Eine zentrale mit der Digitalisierung von Berufsleben und Wissenschaft verbundene Frage ist, inwieweit grundlegende Informatikkenntnisse sowie mathematisch-logische Kenntnisse und Fähigkeiten auch in der Musikwissenschaft für ein zeitgemäßes wissenschaftliches Arbeiten im 21. Jahrhundert unverzichtbar sind und daher in die musikwissenschaftliche Forschung und Lehre zu integrieren sind. Für eine Kognitive Musikwissenschaft, die sowohl musikalische Grundlagenforschung betreibt als auch die epistemologisch-methodologischen Grundlagen der Musikforschung reflektiert, ist dies unabdingbar. Wie sieht es aber generell in einem Fach wie dem der Musikwissenschaft aus, in dem ein historisch-hermeneutisches Selbstverständnis der Disziplin vorherrschend ist? Kann dieses Selbstverständnis im Zuge der epistemologischmethodologischen Veränderungen im Rahmen der Digitalisierung der Wissenschaften uneingeschränkt als die Disziplin maßgeblich bestimmend aufrechterhalten werden?

1 James A. Anderson, After Digital: Computation as Done by Brains and Machines, Oxford 2017. Vgl. ebenfalls Konrad Hinsen, Computation in Science, San Rafael 2015.

2 Joseph E. Aoun, Robot-proof: higher education in the age of artificial intelligence, Cambridge (MA) 2017.

3 Z. B. in der Musikphilologie, vgl. Dörte Schmidt, "Musikwissenschaft", in: Musikleben in Deutschland, hrsg. vom Deutschen Musikrat, Bonn 2019, S. 444-463. 


\title{
Eine epistemologisch-methodologische Anmerkung
}

Wir fokussieren uns auf epistemologisch-methodologische Veränderungen, die sich durch die Digitalisierung für die Musikwissenschaft ergeben. Das in sich selbst versunkene historisierende Denken vergisst allzu gerne seine eigene Historizität und damit die ebenfalls historische Bedingtheit der Idee der Geschichtlichkeit ${ }^{4}$ und bestehender Wissenschaftsklassifikationen, von der insbesondere die dem 19. Jahrhundert entstammende Einteilung in Natur- und Geisteswissenschaften spätestens mit dem Aufkommen der Informationsverarbeitung und Kognitionswissenschaft zu hinterfragen ist, wenn nicht gar als überholt zu gelten hat. Auch waren die im 19. Jahrhundert begonnenen Versuche, eine erkenntnistheoretisch-logische Fundierung der Geistes- bzw. Kulturwissenschaften mittels des Begriffs des Geistes zu erzielen, um ihren Sonderstatus gegenüber den Naturwissenschaften als eine spezifische von den Naturwissenschaften abzugrenzende Wissenschaftskategorie zu begründen, nicht sonderlich erfolgreich. Daher arbeiten die heutigen Geistes- bzw. Kulturwissenschaften - wie die Musikwissenschaft ohne Fundierung und ignorieren gerne epistemologisch-methodologische wie wissenschaftstheoretische Fragen hinsichtlich der Fundierung ihres Faches sowie ihres Gegenstandsbereichs. Es scheint aber, dass dies gerade durch die Digitalisierung und die Kognitionswissenschaft für eine Musikwissenschaft des 21. Jahrhunderts nicht mehr möglich ist. Bernhard Lauth merkt in Bezug auf die Erforschung des Geistes durch die Geisteswissenschaften an:

\begin{abstract}
„Die weit verbreitete methodische Selbstbeschränkung der Geisteswissenschaften auf historische und philologische (,hermeneutische') Methoden hat zur Folge, daß ausgerechnet der Geist selbst und seine kognitiven Fähigkeiten nicht geisteswissenschaftlich, sondern nur naturwissenschaftlich erklärt werden können. Diese methodische Selbstbeschränkung ist aber nicht naturgegeben, sondern ein Resultat von wissenschafts- und philosophiehistorischen Fehlentwicklungen, die ihren Ausgangspunkt im 19. Jahrhundert und hier vor allem in der Philosophie der deutschen Romantik haben. Ein wesentlicher Schritt zur Korrektur dieser Fehlentwicklungen wäre die Rückbesinnung auf den Stellenwert mathematischer Methoden in den Natur- und Geisteswissenschaften. ${ }^{.5}$
\end{abstract}

Mit den Data Sciences sehen auch vermehrt Musikwissenschaftler die Möglichkeit, datenbasierte empirisch-statistische Forschung zu betreiben. Prinzipiell stellt sich allerdings für diese Korpus-basierten Forschungen die Frage nach dem Stellenwert der Anwendung induktivstatistischer Verfahren auf die Musik für eine explanatorische Theoriebildung im Vergleich zu abduktiven Verfahren. ${ }^{6}$ Die adäquate Anwendung mathematischer Methoden setzt jedoch mathematisch-logisches Denken voraus, das innerhalb der Geisteswissenschaften am Gegenstand zu vermitteln wäre. Für die Musikwissenschaft wäre dieser: Musik und Geist. Wie mathematisch-logisches Denken in der Erforschung des Geistes angewandt werden kann, zeigt die Kognitionswissenschaft. Gerade sie, für deren Forschungsparadigma zur Erforschung des

4 Gerhard Bauer, "Geschichtlichkeit” - Wege und Irrwege eines Begriffs, Berlin 1963.

5 Bernhard Lauth, Descartes im Rückspiegel - Der Leib-Seele Dualismus und das naturwissenschaftliche Weltbild, Paderborn 2006, S. 226.

6 Einen Einblick in die aktuelle Diskussion gibt Peter Norvig, "On Chomsky and the two cultures of statistical learning”, <https://sites.tufts.edu/models/files/2019/04/Norvig.pdf> (28. 02.2020). 
Geistes der Begriff Computation ${ }^{7}$ zentral ist, hat die Erforschung des subjektiven Geistes - der menschlichen Kognition - in den letzten Dezennien enorm vorangetrieben. ${ }^{8}$ In der Kognitionswissenschaft zeigt sich besonders gut die Bedeutung der Formalisierung als Mittel der Präzisierung, der Modellbildung und Computersimulation für die wissenschaftliche Erforschung des Geistes. Es ist dabei aber nicht zu verkennen, dass die Kognitionswissenschaft im Kern die Erforschung des objektiven Geistes vernachlässigt. ${ }^{9}$

Durch die Digitalisierung bieten sich für die Musikwissenschaft Chancen und Möglichkeiten für die Erforschung von Musik als geistigem Phänomen - als "musikalische[m] Geist” -, ausgehend vom kognitionswissenschaftlichen Forschungsparadigma und jenseits der Dichotomie von Natur und Geist, "subjektiven" und "objektivierten Geist"10 zu erforschen. Dabei stünde epistemologisch und methodologisch das Konzept der Berechnung (Computation) als Explikat im Zentrum - in Bezug auf den Gegenstand der Musikwissenschaft: Musik und Geist. Es ist jedoch anzumerken, dass durch die klassische Explikation des Berechnungsbegriffs als Turingmaschinen-Berechenbarkeit (Computability) und die damit verbundenen epistemologischmethodologischen Implikationen für wissenschaftliches Forschen ${ }^{11}$, mathematisch-logisches Denken innerhalb dieses aufgespannten formalen Rahmens, als Computational Thinking auszuzeichnen ist.

Die spezifischen Anforderungen an musikwissenschaftliche Forschung und Lehre definieren sich, vom Begriff Computation ausgehend, unserer Auffassung nach daher deutlich breiter und stellen Computational Thinking und Computational Literacy in den Vordergrund.

\section{Computational Thinking und Computational Literacy}

Wir hatten Computational Thinking als eine spezifizierte Form des mathematisch-logischen Denkens ausgewiesen. Dieses Denken erfolgt innerhalb eines algorithmischen Denkrahmens, der durch den Begriff der Turingmaschine in Form von effektiven Verfahren aufgespannt wird. Zu Recht weisen Peter Denning und Matti Tedre ${ }^{12}$ darauf hin, dass Computational Thinking aus wissenschaftshistorischer Sicht auf eine lange Geschichte zurückblicken könne, die mit

7 Clark Glymour, Thinking Things Through: An Introduction to Philosophical Issues and Achievements, 2nd Edition, Cambridge (MA), S. 331-335 gibt eine kurze, einführende Erörterung der mit dem Begriff Computation für kognitionswissenschaftliche Forschungen verbundenen Kernproblematik.

8 The Routledge Handbook of the Computational Mind, hrsg. von Mark Sprevak und Matteo Colombo, New York 2019.

9 Pirmin Stekeler-Weithofer, "Philosophie des Geistes”, in: Enzyklopädie Philosophie und Wissenschaftstheorie, Bd. 6: O-Ra., hrsg. von Jürgen Mittelstraß, Stuttgart 22016, S. 288-289.

10 Nicolai Hartmann, Das Problem des geistigen Seins: Untersuchungen zur Grundlegung der Geschichtsphilosophie und Geisteswissenschaften, Berlin ${ }^{3} 1962$.

11 Volker Beeh, „Der Begriff der Erzeugbarkeit”, in: Germanistische Linguistik 77, H. 1-2 (1977), S. 51-87; legt die Eingrenzung des formalen theoretischen Forschungsrahmens auf effektive Verfahren für Noam Chomskys klassischem Grammatikansatz dar (vgl. insbesondere S. 82-84).

12 Peter Denning und Matti Tedre, Computational thinking, Cambridge (MA) 2019. 
der Antike und dem Entstehen der (beweisenden) Mathematik beginne. Ihnen zufolge lasse sich Computational Thinking zunächst fernab tatsächlicher Rechnerimplementierungen als grundlegende Kompetenz ausweisen, Probleme als berechenbare Prozesse auszudrücken (designing) und die uns umgebende Welt als Komplex von Informationsverarbeitungsprozessen zu erklären (explaining). ${ }^{13}$ Im Bereich datengestützter, rechnerbasierter Musikforschung dürften hierbei nach unserer Auffassung zunächst die konzeptionelle Ebene sowie die Vermittlung grundlegender algorithmischer Methoden in Form von vorliegenden und zu entwerfenden, problembezogenen Algorithmen von besonderem Interesse sein. Hierbei ist trotz aller Nähe zur Implementierung zunächst die Beobachtung zentral, dass Computational Thinking nicht unmittelbar in die Kompetenz eines Subjektes übersetzt werden kann, funktionalen Programmcode zu implementieren, oder gar computerähnliches Denken zu erlernen.

Die Befähigung zu Computational Thinking impliziert zunächst konzeptionelle Kompetenzen, welche sich in den Teilkompetenzen der Dekomposition, Abstraktion und Repräsentation widerspiegeln. Die Fähigkeit zur Dekomposition eines gegebenen Problems bezeichnet hierbei die Kompetenz, Teilprozesse zu seiner Lösung zu identifizieren und modularisiert zu formalisieren. Abstraktion meint in diesem Kontext das Vermögen, gezielt Eigenheiten der Realisation eines komplexen Systems oder Problems auszublenden, um zu einem allgemeineren und verallgemeinerbaren Verständnis seiner inneren Funktionsweise durchzudringen. Bei der computationalen Behandlung gegebener Probleme stellt sich zuletzt die Frage nach einer adäquaten formalen Repräsentation beschriebener Phänomene.

Von diesen konzeptionellen Kompetenzen sind die Methodenkompetenzen zu unterscheiden, welche zu einer faktischen Implementierung eines entworfenen Systems befähigen. Diese sind auch zu verbinden mit der Frage nach der Automatisierbarkeit einer gegebenen Problemlösung.

Es ist davon auszugehen, dass bei einer entsprechenden Ausbalancierung beider Kompetenzbereiche entlang der von Denning und Tedre ${ }^{14}$ gewünschten Wechselwirkung von "science" und "engineering" starke Synergieeffekte zu erwarten sind, weshalb sich eine entsprechende Beförderung von Computational Thinking in der universitären Bildung beiden Gebieten angemessen in modifizierter Form widmen müssen wird.

Es ist darauf hinzuweisen, dass Denning und Tedre bei ihren Erörterungen die Geisteswissenschaften nicht im Blick haben. Für die Vermittlung der Programmierfähigkeit im Kontext der Geisteswissenschaften scheint es allerdings angebracht, Programmieren nicht rein ingenieurwissenschaftlich zu verstehen. Aus geisteswissenschaftlicher Perspektive betrachtet, knüpft das Programmieren an die traditionellen Lese- und Schreibfähigkeiten an - aber erweitert diese. Es kann von Computational Literacy gesprochen werden, da das Lesen und Schreiben von Computerprogrammen - wie das Lesen und Schreiben von Texten - eine generelle, sozial

13 Ebd., S.4.

14 Denning/Tedre. Computational thinking, S. 101-103. 
erforderliche Fähigkeit darstellt. Annette Vee hat den Ausdruck "computational literacy" benutzt und versteht unter "literacy" "[...] a widely held ability to compose and interpret symbolic and communicative texts in an infrastructural medium. " 15 Die Computational Literacy "[...] links the theoretical apparatus with the computation that is central to computer programming." 16 Vee bestimmt "computational literacy" als "[...] the constellation of abilities to break a complex process down into small procedures and then express - or , write'- those procedures using the technology of code that may be ,read' by a non-human entity such as a computer. ${ }^{\prime 17}$

Computational Literacy steht also in enger Verbindung zum Computational Thinking. Sie unterscheidet sich aber dadurch, dass sie zum einen an das Schreiben und Lesen von Code gekoppelt ist. Zum anderen hebt sie aber das Lesen und Schreiben von Code als eine über eine spezielle Disziplin hinausgehende, generelle soziale Fähigkeit hervor. Computational Literacy deutet zugleich darauf hin, dass der Computer nicht nur ein technologisches Artefakt, sondern auch ein soziales Konstrukt darstellt. ${ }^{18}$ Computational Thinking ist dagegen nicht direkt an das Erstellen von Code gekoppelt. Es ist aber eine besonders ausgezeichnete Form des mathematisch-logischen Denkens, das mit der Digitalisierung auch außerhalb der Wissenschaft in Gesellschaft und Wirtschaft an Bedeutung gewinnt. Computational Thinking und Computational Literacy stehen also in wechselseitiger Beziehung. Will man nun diese Denkstile in der universitären Ausbildung vermitteln, so stellt sich die Frage nach einem hochschulpädagogischen Ansatz.

\section{Der pädagogische Ansatz: Konstruktivismus und situatives Lernen}

Der von uns präferierte hochschulpädagogische Ansatz erfolgt im Rahmen des konstruktiven und situativen Lernens. Der pädagogische Konstruktivismus geht im Kern davon aus, dass das Aneignen eines Wissensbereiches durch aktiv-explorative Tätigkeiten des Lernenden erfolgt und der Lehrende in der Lehr-/Lernsituation zu einem großen Teil die Rolle eines Facilitators einnimmt. Das situative Lernen dagegen berücksichtigt den Kontext und die sozialen Bedingungen des Lernens. Die Grundideen des situativen Lernens berücksichtigend, gehen wir davon aus, dass die Bereitstellung einer computationalen Lern- und Arbeitsumgebung als digitalem Habitat ${ }^{19}$ eine gute und natürliche Ausgangsbasis darstellt, um ein computationales Umfeld zu erzeugen. In diesem digitalen Habitat können sich Lernende in verschiedene Wissensbereiche

15 Annette Vee, „Understanding Programming as a Literacy”, in: Literacy in Composition Studies 1, H. 2 (2013), S. 42-64, hier S. 46, <http://licsjournal.org/OJS/index.php/LiCS/article/view/24/26> (27.02.2020).

16 Ebd., S. 46.

17 Ebd., S. 47.

18 Seymour Papert, "Information Technology and Education: Computer Criticism vs. Technocentric Thinking”, in: M.I.T. Media Lab Epistemology and Learning Memo. No. 1, Cambridge (MA) 1990.

19 Etienne Wenger, Nancy White und John D. Smith, Digital Habitats: stewarding technology for communities, Portland 2009. 
der Musikwissenschaft einarbeiten und dabei grundlegende Kenntnisse und Fertigkeiten aus dem Bereich der Informatik aneignen, indem die Lernenden in natürlicher Umgebung Computational Thinking und Computational Literacy praktizieren und dabei - ähnlich einem biologischen Habitat - ihre digitale Umwelt fortwährend verändern und gestalten. Ein solches sich ständig veränderndes digitales Habitat bietet zugleich auch die Möglichkeit, die durch technologische Entwicklungen bedingten rasanten Veränderungen zu integrieren und zugleich Lernen des Lernens zu praktizieren.

Aus dem bisher Gesagten ergibt sich, dass Computational Thinking und Literacy für eine Musikwissenschaft des 21. Jahrhunderts grundlegend ist und dass das kulturwissenschaftlich historisch-hermeneutische Denken in der Musikwissenschaft durch dieses zu komplementieren ist, indem es in die Lehre einbezogen wird.

\section{Ein digitales Habitat für musikwissenschaftliche Lehre und Forschung: Jupyter Notebook}

Eine hiervon ausgehende Einführung für Studierende geisteswissenschaftlicher Disziplinen, welche zugleich auf den Erwerb grundlegender Kompetenzen des Computational Thinking und der Computational Literacy abzielt, steht also konzeptionell vor der Herausforderung, einerseits allgemeine Konzepte und Methoden der Informatik adäquat und zugänglich zu vermitteln und diese andererseits auf intradisziplinär relevante Fragestellungen und Themengebiete beziehen zu können. ${ }^{20}$

Eines der Kernanliegen des vorliegenden Projektes besteht darin, diese schwierige Ausgangssituation für die Lehre technisch und medial bestmöglich ,vorzuentlasten' und ein digitales Habitat zur Verfügung zu stellen. Aus diesem Grund setzt es auf die in der Forschung bereits seit Jahren weit verbreitete Architektur Jupyter Notebook als Lern- und Forschungsumgebung. Die weite Verbreitung von Jupyter Notebook lässt sich u. a. anhand einer Erhebung der Anzahl frei zugänglicher Notebook-Dateien für IPython (.ipynb) beziffern. Nach Jeffrey Perkel ist zu beobachten, dass die Anzahl von Jupyter Notebooks in öffentlich einsichtigen GitHub-Repositorien gegenüber 20.000 im Jahr 2015 bereits auf über 2,5 Millionen im Jahr 2018 angestiegen ist. ${ }^{21} \mathrm{Zu}$ einer besseren Einordnung der Popularität von Jupyter Notebook ist es hilfreich, sich zunächst einige grundlegende Gedanken über die Programmiersprache Python zu machen, um sich im Anschluss historisch die Entwicklung zum gegenwärtigen Stand des JupyterlabFrontends zu vergegenwärtigen.

20 Brian Kokensparger, Guide to Programming for the Digital Humanities: Lessons for Introductory Python, Cham 2018.

21 Jeffrey M. Perkel, "Why Jupyter is data scientists' computational notebook of choice”, in: Nature 563 (2018), S. 145-147, <https://www.nature.com/articles/d41586-018-07196-1> (27.10.2020). 


\section{Jupyter Notebook: eine kurze Historie}

Python hat im Bereich der Data Sciences in den letzten Jahren zunehmende Verbreitung erfahren und dient nicht zuletzt auf Grund seiner Nutzbarmachung in Form von interaktiven Publikationsformaten als weitläufig rezipierter Standard. ${ }^{22}$ Python lässt sich als highlevel, objektorientierte Programmiersprache ausweisen, welche auf Grund ihres Open-Source Charakters eine sehr hohe Flexibilität hinsichtlich ihrer möglichen Anwendungsgebiete aufweist: „Python is a general-purpose programming language that can be used effectively to build almost any kind of program that does not need direct access to the computer's hardware. "23 Python ist eine interpretierte Programmiersprache, welche im Gegensatz zu kompilierten Programmiersprachen eine unmittelbare Auswertung der im Code enthaltenen Ausdrücke und Anweisungen ermöglicht. Dieses letztgenannte Spezifikum ermöglicht eine direktere Einflussnahme des Programmierenden auf den Programmcode sowie die Manipulation von Teilcode ohne Kompilierung, wodurch letztlich interaktive Programmierung ermöglicht wird. 2001 wurde IPython als Adaption des Pythoninterpreters entwickelt, welche in einem interaktiveren Format die Programmiersprache zunächst für die neurowissenschaftliche Forschung handhabbar gemacht hat. ${ }^{24} 2011$ folgte die Einführung des IPython Notebooks als webbasiertes Interface für die Python-Programmierung. 2014 entwickelte sich aus dem IPython Projekt das Project Juypter, welches auf Open-Source Basis den Kerngedanken der IPython-Initiative auf die namensgebenden Programmiersprachen Julia, Python und $R$ erweiterte und das Jupyter Notebook etablierte. Das Jupyter Notebook hat sich seitdem zu einem regelrechten Ökosystem entwickelt, das flexibel erweiterbar ist. Das zentrale Anliegen des Project Jupyter, welches einer transparenten Entwicklung unterliegt und über GitHub auch die Community mit in die Weiterentwicklung einbezieht, ist die Beförderung interaktiver Data Science. ${ }^{25}$

\section{Jupyter Notebook: die elementare Struktur}

Jupyter Notebook ${ }^{26}$ verfügt über zwei graphische Benutzerschnittstellen: Jupyter Classic und die neuere, in unserem Projekt verwendete Schnittstelle Jupyterlab, deren Oberfläche sich in ihrer Funktionalität als flexibel erweiterbar erweist ${ }^{27}$ - beispielsweise in Form der Konvertierung

22 Cyrille Rossant, IPython Interactive Computing and Visualization Cookbook: Over 100 hands-on recipes to sharpen your skills in high-performance numerical computing and data science in the Jupyter Notebook, Birmingham 2018.

23 John V. Guttag, Introduction to computation and programming using Python: With application to understanding data, Cambridge 22016, S. 8.

24 Vgl. hierzu und im Folgenden Rossant, IPython Interactive Computing.

25 Vgl. „About us”, in: Jupyter, <https://jupyter.org/about> (06.04.2020).

26 Vgl. für Details zu Jupyter Notebook JupyterLab Quick Start Guide: A beginner's guide to the next-gen, web-based interactive computing environment for data science, hrsg. von Lindsay Richman et al., Birmingham 2019.

27 Einen Überblick über die Vorteile von Jupyterlab bietet Rene Draschwandtner, „JupyterLab - A Next Gen Python Data Science IDE. An Article Nudging Data Scientists towards JupyterLab", in: Towards Data Science, <https:// towardsdatascience.com/jupyterlab-a-next-gen-python-data-science-ide-562d216b023d> (27.02.2020). 
von Notebooks in gängige Dateiformate wie PDF oder RTF sowie Präsentationen mit lauffähigem Programmcode. Jupyter Notebook kann lokal und als Server installiert werden. Mittels Jupyterhub kann ein Jupyter-Server eingerichtet werden, der es erlaubt, dass mehrere Personen zugleich Zugriff haben und gemeinsam an Dateien gearbeitet werden kann. Die Zugänglichkeit von Jupyter Notebook in der Lehre wird durch den Einsatz von Jupyterhub erleichtert, wodurch der Aufwand bei der Einrichtung adäquater Arbeitsumgebungen gänzlich serverseitig aufgefangen werden kann und es den Lehrenden und Studierenden ermöglicht wird, sich über ein einfaches Browserinterface mit einer funktionstüchtigen Umgebung zu verbinden.

\section{Jupyter Notebook: die Funktionalität}

Jupyter Notebook ermöglicht die interaktive Texterstellung und Ausführung von Programmcode u. a. in den Programmiersprachen Python, Julia und R. Ein einzelnes Notebook besteht aus aktiven Codezellen für die Programmierung und Textzellen, mit in Markdown, HTML oder LaTeX aufbereitetem Hypertext. Diese Struktur ermöglicht neben der Ausführung von partiellem Code - während der Erstellung eines Notebooks - die Kommentierung und Ausarbeitung der enthaltenen Konzepte in einem Dokument, wodurch traditionelle Möglichkeiten der Präsentation und Diskussion von Programmcode und Wissensbereichen in separaten Begleittexten signifikant erweitert werden.

Das digitale Habitat wird im Rahmen unseres Projektes mit Hilfe eines Jupyterhubs bereitgestellt. Hierdurch wird auf die zentrale Verwaltung individueller Arbeitsbereiche für Lehrende und Studierende gesetzt, welche über jedes internetfähige Gerät mit Webbrowser zugänglich sind. Dies ermöglicht es, das gesamte digitale Habitat über einen Webserver zu administrieren. Jupyterhub reduziert somit auch Probleme, welche sich aus der lokalen Einrichtung von Arbeitsumgebungen auf der Hardware von Lehrenden und Lernenden ergeben und welche in der Vergangenheit eine wesentliche Hürde in der Vermittlung rechnerbasierter Methodenkompetenzen dargestellt haben.

Das digitale Habitat kann in Seminarkontexten als digitaler Raum den materiellen und sozialen Raum der Präsenzkurse erweitern und somit einen hybriden Lehr-/Lernraum im Sinne eines Third Space ${ }^{28}$ konstituieren oder auch selbstständig in Form reiner Internetkurse eingesetzt werden. Es ermöglicht ein umfassendes E-Learning-Angebot, welches beispielsweise die Bereitstellung von Kursmaterialien, relevanten Datensätzen für Analysevorhaben und virtuellen Klassenräumen samt individualisierten Arbeitsaufträgen umfasst. Außerdem ermöglicht es im Zusammenspiel mit traditionellen Seminarstrukturen die Umsetzung von Strategien des Blended Learning.

28 Eva-Christina Edinger und Ricarda T. D. Reimer, „Thirdspace als hybride Lernumgebung. Die Kombination materieller und virtueller Lernräume", in: Erwachsenenbildung und Raum. Theorie und Praxis der Erwachsenenbildung, hrsg. von Christian Bernhard et al., Bielefeld 2015, S. 205-216. 


\section{Jupyter Notebook: Lehrerfahrungen}

Im digitalen Habitat wurden grundlegende Einführungen in Methoden und Konzepte der Computational Musicology gelehrt und Methodenkompetenzen u. a. für die digitale Korpusforschung in Python und R vermittelt. Aus der Perspektive der rechnerbasierten Musikforschung wurden aktuelle, interdisziplinäre Forschungsdiskurse behandelt, darunter mathematische Aspekte antiker, mittelalterlicher und chinesischer Musiktheorie - beispielsweise Tonsysteme und Intervalllehre -, Computational Modeling, Musik und Künstliche Intelligenz, Artifical Neural Networks sowie rechnerbasierte musikalische Analyse unter Zuhilfenahme von Music21 zur Verarbeitung symbolisch repräsentierter Musik. ${ }^{29}$ Darüber hinaus wurden im Rahmen verschiedener Seminare die Möglichkeiten individualisierter Lernmöglichkeiten im digitalen Habitat erprobt und die didaktischen Konsequenzen der Arbeit mit der Jupyter-NotebookUmgebung reflektiert. Hierdurch liegen Erfahrungen vor, die in die Weiterentwicklung dieses digitalen Lehr-/Lernkonzeptes einfließen.

\section{Zusammenfassung}

Wir haben argumentiert, dass die Digitalisierung für die Musikwissenschaft nicht nur hinsichtlich der informationstechnologischen Infrastruktur eine Herausforderung birgt, sondern dass auf tieferer epistemologisch-methodologischer Ebene eine Integration mathematisch-logischen Denkens für eine Musikwissenschaft des 21. Jahrhunderts erforderlich ist. Dieses Denken wird in Verbindung mit der Digitalisierung als Computational Thinking und Computational Literacy charakterisiert. Computational Thinking fokussiert sich auf den von der faktischen Programmierung losgelösten Denkstil, während Computational Literacy die Notwendigkeit der Programmierung als generelle, sozial relevante Kompetenz betont. Wir gehen davon aus, dass die Realisierung eines digitalen Habitats die beste Voraussetzung für die Vermittlung dieses Denkens darstellt. Zugleich steht diese Idee im Einklang mit dem pädagogischen Konstruktivismus und dem situierten Lernen. Als technologische Realisierung eines digitalen Habitats für die musikwissenschaftliche Lehre und Forschung nutzen wir Jupyter Notebook, bereitgestellt über Jupyterhub. Wir haben diverse Kurse wie z. B. zur grundlegenden Programmierung in Python und R, Computational Music Theory, digitaler Korpusforschung, rechnergestützter Komposition mit KI-Systemen sowie der Modellierung mit Künstlichen Neuronalen Netzen durchgeführt. Inzwischen können wir auf zwei Jahre Lehr-/Lernerfahrung im digitalen Habitat zurückschauen und feststellen, dass sich der Einsatz prinzipiell bewährt hat und auf stetig zunehmendes Interesse der Studierenden stößt, so dass nach und nach eine Integration von

29 Michael Scott Cuthbert und Christopher T. Ariza, C., „music21: A Toolkit for Computer-Aided Musicology and Symbolic Music Data", in: 11th International Society for Music Information Retrieval Conference (ISMIR 2010), August 9-13, 2010, Utrecht, Netherlands, hrsg. von J. Stephen Downie und Remco C. Veltkamp, Utrecht 2010, S. 637-642. 
Computational Thinking und Computational Literacy in die Musikwissenschaft erfolgt. Grundsätzlich bleibt für die Digitalisierung in der Musikwissenschaft festzuhalten: Auch ohne die angesprochenen epistemologisch-methodologischen Implikationen gilt für die Auswirkungen der Digitalisierung in Wissenschaft, Gesellschaft und Wirtschaft spätestens im 21. Jahrhundert auch für die Musikwissenschaft die bereits 35 Jahre alte Aussage John Kemenys, in der im Sinne Vees $^{30}$ "computer" durch "computational” zu ersetzen wäre: "Someday computer literacy will be a condition for employment, possibly for survival [...]."

Zitation: Uwe Seifert, Sebastian Klaßmann, Timo Varelmann und Nils Dahmen, "Computational Thinking in der Musikwissenschaft: Jupyter Notebook als Umgebung für Lehre und Forschung", in: Freie Beiträge zur Jahrestagung der Gesellschaft für Musikforschung 2019, hrsg. von Nina Jaeschke und Rebecca Grotjahn (= Musikwissenschaft: Aktuelle Perspektiven. Bericht über die Jahrestagung der Gesellschaft für Musikforschung 2019 in Paderborn und Detmold, Bd. 1), Detmold 2020, S. 309-319, DOI: 10.25366/2020.72.

30 Vee, Understanding Programming, S. 47. 


\section{Abstract}

We show that in connection with the digitalization of musicology a special kind of mathematical and logical thinking, i. e. computational thinking/literacy, is in need. Computational thinking is characterized by effective procedures whereas computational literacy includes the implementation of these procedures on machines, i.e. programming. Both are the core of formalization, model building and computer simulation. Furthermore, we point out that "computation" as a central concept for the sciences in the $21^{\text {st }}$ century and its use in cognitive science and the computational sciences make it necessary to reassess the basic assumptions underlying musicological research as science of mind (Geisteswissenschaft). We propose a digital habitat to integrate computational thinking/literacy in musicology and to become acquainted with model building and computer simulation. Jupyter Notebook provides a basis for such a digital habitat. We describe our use of Jupyter Notebook as a teaching environment for computational thinking/literacy.

\section{Kurzvitae}

Uwe Seifert ist Professor für Musikwissenschaft mit Schwerpunkt Systematische Musikwissenschaft am Musikwissenschaftlichen Institut der Universität zu Köln. Er betreibt Kognitive Musikwissenschaft als musikalische Grundlagenforschung im kognitionswissenschaftlichen Forschungsparadigma. Einen seiner aktuellen Forschungsschwerpunkte bildet die Evolution der menschlichen Musikfähigkeit, die er, vom Forschungsrahmen der Spiegelsystemhypothese ausgehend, unter Rekurs auf neuroethologische Forschungen untersucht.

Sebastian Klaßmann unterrichtet Computational Musicology und ist Jupyter-Systemadministrator am Musikwissenschaftlichen Institut der Universität zu Köln. Nach Abschluss seines Jazzstudiums in Rotterdam (2014) und des Masterstudiums der Musikwissenschaft und Religion/ Kultur/Moderne in Köln (2018) widmet er sich in seinem Dissertationsprojekt der computationalen Musiktheorie. Zudem ist er als Instrumentalpädagoge tätig.

Timo Varelmann schloss 2016 sein Masterstudium der Musikwissenschaft und Linguistik/ Phonetik an der Universität zu Köln ab und unterrichtete als Lehrbeauftragter Grundlagen der Statistik und der statistischen Programmierung in $\mathrm{R}$ für Musikwissenschaften. Derzeit ist er Doktorand am Musikwissenschaftlichen Institut der Universität zu Köln und Mitarbeiter des Deutschen Musikinformationszentrums.

Nils Dahmen ist studentische Hilfskraft am Musikwissenschaftlichen Institut der Universität zu Köln. Er ist Student der Musikwissenschaft und Philosophie. Seit 2018 ist er Mitglied einer die anderen Autoren einschließenden Arbeitsgruppe zum Thema computationelle Forschung in Musik- und Geisteswissenschaften. Machine Learning und mathematische Methoden bilden seinen inhaltlichen Fokus. 


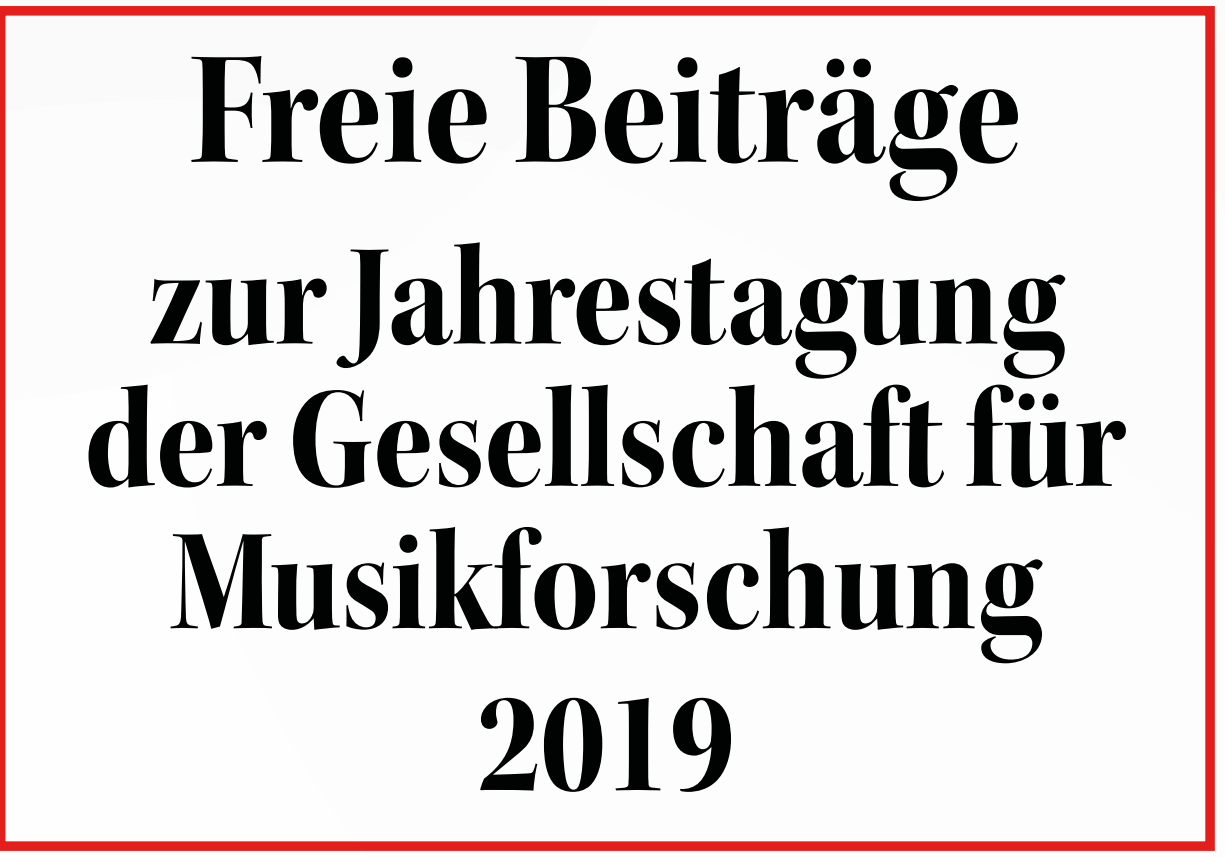

Herausgegeben von Nina Jaeschke und Rebecca Grotjahn

Musikwissenschaft: Aktuelle Perspektiven 1 
Freie Beiträge 


\section{Musikwissenschaft: Aktuelle Perspektiven}

Bericht über die Jahrestagung der Gesellschaft für Musikforschung 2019 in Paderborn und Detmold

Herausgegeben von Rebecca Grotjahn und Nina Jaeschke

Band 1 


\section{Freie Beiträge}

\section{zur Jahrestagung der Gesellschaft für Musikforschung 2019}

Herausgegeben von Nina Jaeschke und Rebecca Grotjahn

Detmold: Musikwissenschaftliches Seminar der Universität Paderborn und der Hochschule für Musik Detmold 2020 
DOI: $10.25366 / 2020.42$

Online-Version verfügbar unter der Lizenz: Urheberrecht 1.0, $<$ https://rightsstatements.org/page/InC/1.0/?language=de>

Bibliografische Information der Deutschen Nationalbibliothek

Die Deutsche Nationalbibliothek verzeichnet diese Publikation in der Deutschen Nationalbibliografie; detaillierte bibliografische Daten sind im Internet über http://dnb.d-nb.de abrufbar.

\section{Impressum}

Redaktion: Nina Jaeschke, Rebecca Grotjahn und Jonas Spieker Satz: Nina Jaeschke

(C) Musikwissenschaftliches Seminar der Universität Paderborn und der Hochschule für Musik Detmold 2020 


\section{INHALT}

Vorwort $\quad$ IX

Komponieren für das Radio: Akteure, Diskurse, Praktiken $\quad 1$

Musikwissenschaft - Feminismus - Kritik: Ein Generationenaustausch 6

\section{Stefan Alschner}

Der Wagner-Sänger Joseph Aloys Tichatschek - Vom Nachlass zum Netzwerk

\section{Alenka Barber-Kersovan}

Songs for the Goddess. Das popmusikalische Neo-Matriarchat zwischen Ethno-Beat,

erfundenen Traditionen und kommerzieller Vermarktung

Elias Berner, Julia Jaklin, Peter Provaznik, Matej Santi, Cornelia Szabó-Knotik

Musikgeschichte anders erzählen? Das Beispiel der 1970er in Österreich.

Musikhistoriographie in der Zeit der Digitalisierung

\section{Mauro Fosco Bertola}

„Ein Laut so klagevoll”. Lohengrin zwischen Richard Wagner und Salvatore Sciarrino

\section{Matthieu Cailliez}

Europäische Rezeption der Berliner Hofoper und Hofkapelle von 1842 bis 1849

\section{lacopo Cividini}

Zwischen klassischer Musikphilologie und angewandter Informatik:

Die Digitale Mozart-Edition (DME) der Stiftung Mozarteum Salzburg

\section{Marko Deisinger}

Fortschrittliche Technologie im Dienste eines Antimodernisten.

Heinrich Schenker und der österreichische Rundfunk

\section{Norbert Dubowy}

Vom Kritischen Bericht zur Kritischen Dokumentation am Beispiel der Digital-interaktiven Mozart-Edition

\section{Markus Engelhardt}

Musik zwischen Nation Building und Internationalität. Italien um 1900

\section{Maryam Haiawi}

Das Oratorium im Spannungsfeld der Konfessionen: 


\section{Judith I. Haug}

"Manch eine*r liegt, morgens noch trunken, im Rosengarten" - Rekonstruktionen

osmanischer Musikgeschichte in Gesangstextsammlungen

\section{Renate Koch}

Marcel Prawy und das erste Broadway-Musical im Österreich der Nachkriegszeit

Susanne Kogler, Julia Mair, Juliane Oberegger, Johanna Trummer

Erich Marckhl - Musikausbildung in der Steiermark nach 1945.

Brüche und Kontinuitäten

\section{Marie-Anne Kohl}

Die weinende Jury. "Geschlechtslose" Tränen bei globalen Musik-Castingshows?

\section{Fabian Kolb}

Tanztheater und filmische Ästhetik. Cineastische Einflüsse und Gestaltungsweisen in den Kompositionen für die Ballets Suédois 1920-1925

\section{Christian Lehmann}

Tempobezeichnungen von Julius Stockhausen für Die schöne Müllerin:

Ein Quellenfund

\section{Martin Link}

Signum et gens - Zur Gendersemiotik in Clara und Robert Schumanns Liederzyklus Liebesfrühling

\section{Livio Marcaletti}

„Strafspiel" und satirische Stilmittel in musikdramatischen Gattungen des frühen 18. Jahrhunderts

\section{Tobias Marx, Martin Lissner}

Thüringer Musikszene - Jugendmusikredaktionen als außerschulische musikbezogene Bildungskontexte

\section{Maho Naito}

Die Parallelität der Entstehungsprozesse der ersten beiden Symphonien Gustav

Mahlers: Instrumentation, Revision und Dirigierpraxis

\section{Elisa Novara}

Eine Schumann-Werkstatt? Zur Übertragbarkeit der Methoden vom Projekt 
Theodora Oancea, Joachim Pollmann, Jonas Spieker

Kollaborateure - Involvierte - Profiteure. Erarbeitung eines Online-Lexikons zur

Musik in der NS-Zeit

\section{Kiron Patka}

„Ich wollte eigentlich Sängerin werden." Berufsselbstbilder von Tontechniker*innen im Radio

\section{Siegwart Reichwald}

Die Leiden der jungen Clara: Das Klaviertrio Opus 17 als Ausdruck einer Neu-

Romantikerin

\section{Elisa Ringendahl}

Lied versus Oper - Pole musikalischer Gattungen bei Oscar Bie

\section{Benedikt Schubert}

Struktur und Exegese. Über Eigentümlichkeiten in der Arie "Des Vaters Stimme ließ sich hören" (BWV 7/4)

Uwe Seifert, Sebastian Klaßmann, Timo Varelmann, Nils Dahmen

Computational Thinking in der Musikwissenschaft: Jupyter Notebook als Umgebung

für Lehre und Forschung

\section{Yusuke Takamatsu}

Synthese als Modus der Prozessualität bei Schubert:

Sein spezifisches Wiederholungsprinzip im langsamen Satz

\section{Daniel Tiemeyer}

Johann Nepomuk Hummels Sonate in fis-Moll Op. 81 - Studien zu Entstehungs-

hintergrund, Rezeption und formalerStruktur

\section{Andrea van der Smissen}

Musikalische Innovation im Umfeld der Moderne und historischen Avantgarde in Ungarn

\section{Tim Ziemer, Holger Schultheis}

Psychoakustische Sonifikation zur Navigation in bildgeführter Chirurgie

\section{Magdalena Zorn}

Musik mit dem Radio hören: Über den Begriff der musikalischen Aufführung 
Gabriele Buschmeier in memoriam 


\section{Vorwort}

Die vorliegenden Bände dokumentieren die Jahrestagung der Gesellschaft für Musikforschung 2019. In den dreieinhalb Tagen vom 23. bis zum 26. September 2019 wurden in Paderborn und Detmold nicht weniger als 185 Beiträge präsentiert, verteilt auf diverse Symposien, Round tables, Freie Sektionen und Postersessions. Sie alle auf einen Nenner bringen zu wollen, ist ein Ding der Unmöglichkeit - und das ist gut so, ist es doch Ziel der Jahrestagungen, die große Vielfalt der Themen und Methoden des Faches Musikwissenschaft abzubilden. Um die thematische Vielfalt der freien Referate angemessen abbilden zu können und gleichzeitig den inhaltlichen Schwerpunkten der beiden hier publizierten Hauptsymposien ausreichend Raum bieten zu können, erscheinen diese in drei Bänden.

„Musikwissenschaft: Aktuelle Perspektiven": Der Titel der kleinen Reihe ist keine Verlegenheitslösung. Musikwissenschaft im Kontext der Digital Humanities; Musikwissenschaft und Feminismus; Musik und Medien; Musikalische Interpretation - schon die Felder, die von den vier Hauptsymposien bespielt wurden, wären noch vor wenigen Jahrzehnten allenfalls an der Peripherie das Faches zu finden gewesen. Sie entsprechen Arbeitsschwerpunkten der Lehrenden am Musikwissenschaftlichen Seminar der Universität Paderborn und der Hochschule für Musik Detmold, das die Tagung ausrichtete. Zugleich nehmen sie Bezug auf aktuelle Ereignisse und Entwicklungen. So erwuchs das von Andreas Münzmay und Joachim Veit organisierte Symposium „Brückenschläge - Informatik und Musikwissenschaft im Dialog" unmittelbar aus den Erfahrungen im Virtuellen Forschungsverbund Edirom (ViFE) und im fakultäten- und hochschulübergreifenden Zentrum Musik-Edition-Medien (ZenMEM). Der 200. Geburtstag von Clara Wieck/Schumann war der Anlass für das von Rebecca Grotjahn geleitete Symposium „Die Begleiterin - Clara Schumann, Lied und Liedinterpretation", das in enger Kooperation mit der Hochschule für Musik Detmold durchgeführt wurde. Das Hauptsymposium „Brückenschläge" wird in einem separaten Band publiziert (Bd. 3 der vorliegenden Reihe). Im Rahmen dieses Symposiums führte die von Stefanie Acquavella-Rauch geleitete Fachgruppe Digitale Musikwissenschaft eine Posterpräsentation durch, die von den Beiträger*innen erfreulicherweise zu kürzeren Texten umgearbeitet wurden, sodass sie hier ebenfalls, zusammen mit den Postern,

publiziert werden können. Hinzu kommen einige Beiträge, die bereits bei der Jahrestagung 2018 in Osnabrück präsentiert wurden. Auch das Hauptsymposium "Die Begleiterin" wird in einem eigenen Band (Bd. 2) publiziert. Die Beiträge zu den beiden anderen Hauptsymposien hingegen werden an anderen Orten veröffentlicht; in Band 1 („Freie Beiträge zur Jahrestagung der Gesellschaft für Musikforschung 2019") der vorliegenden Publikation finden sich jedoch Einführungen und Abstracts. Das Symposium „Komponieren für das Radio" unter Leitung von Antje Tumat und Camilla Bork (Katholieke Universiteit Leuven) behandelte Einflüsse des Mediums auf Kompositionsprozesse sowie durch radiophone Kompositionen bzw. radiophonen Klang ausgelöste Diskurse. Sarah Schauberger und Cornelia Bartsch (Universität Oldenburg) nahmen das 25-jährige Jubiläum der Fachgruppe Frauen- und Genderstudien zum Anlass für einen Generationenaustausch zum Thema "Musikwissenschaft - Feminismus - Kritik": Was wa- 
ren vor einem Vierteljahrhundert Inhalte und Aufgaben einer feministischen Musikwissenschaft und wie kann sich diese heute positionieren?

Bewusst haben wir im Tagungsbericht auf inhaltliche Eingriffe in die Beiträge verzichtet. ${ }^{1}$ Das gilt besonders für die Freien Referate: Es galt, den Charakter der Jahrestagung als Forum für ,freie', d. h. innovative und auch experimentelle Gedanken zu wahren. Einige Kolleg*innen, die die Tagung mit Vorträgen und Posterpräsentationen bereichert hatten, haben sich gegen eine Publikation im vorliegenden Band entschieden - sei es, weil sie eine Möglichkeit fanden, ihre Beiträge in einem inhaltlich passenderen Rahmen zu veröffentlichen, sei es, weil ihre Überlegungen in ihre entstehenden Qualifikationsschriften fließen sollen, oder sei es, weil sie von den Autor*innen in der vorgetragenen Form zunächst verworfen wurden. Auch damit erfüllt eine Freie-Referate-Sektion ihren Zweck: Die Diskussionen mit der versammelten Fach-Öffentlichkeit sollen dabei helfen, Gedanken weiterzuentwickeln und zu verändern. In diesem Sinne sei allen Beteiligten - den Autor*innen, den nichtpublizierenden Referent*innen und den Mit-Diskutant*innen - ganz herzlich gedankt für ihr Mitwirken bei der Tagung.

Unser herzlicher Dank gilt einer Reihe weiterer Personen, die zum Gelingen dieser drei Bände beigetragen haben. Hier ist besonders Jonas Spieker zu nennen, der uns tatkräftig bei der Redaktion geholfen hat. Andrea Hammes (SLUB Dresden) sei herzlich für die Aufnahme unseres Bandes auf musiconn.publish gedankt - wir freuen uns, damit unsererseits zur Etablierung dieser innovativen Publikationsplattform beizutragen.

Erneut möchten wir an dieser Stelle allen Menschen danken, die uns bei der Organisation, Ausrichtung und Finanzierung der Tagung selbst unterstützt haben: der Präsidentin der Universität Paderborn, Prof. Dr. Birgitt Riegraf, dem Rektor der Hochschule für Musik Detmold, Prof. Dr. Thomas Grosse, den Kolleginnen und Kollegen der beiden beteiligten Hochschulen, dem Vorstand der Gesellschaft für Musikforschung, der Universitätsgesellschaft Paderborn und allen Sponsoren. Besonders dankbar sind wir den Mitarbeiter*innen und den studentischen bzw. wissenschaftlichen Hilfskräften des Musikwissenschaftlichen Seminars, die bei der Vorbereitung und Ausrichtung der Tagung immensen Einsatz zeigten - stellvertretend sei an dieser Stelle Johanna Imm erwähnt, die zusammen mit Nina Jaeschke das Herz des Organisationsteams bildete.

Wir widmen diese Reihe Dr. Gabriele Buschmeier, dem langjährigen Vorstandsmitglied der Gesellschaft für Musikforschung, die kurz vor der Publikation dieses Bandes unerwartet verstarb.

Detmold, im September 2020

Rebecca Grotjahn und Nina Jaeschke

Zitation: Rebecca Grotjahn und Nina Jaeschke, „Vorwort”, in: Freie Beiträge zur Jahrestagung der Gesellschaft für Musikforschung 2019, hrsg. von Nina Jaeschke und Rebecca Grotjahn (= Musikwissenschaft: Aktuelle Perspektiven. Bericht über die Jahrestagung der Gesellschaft für Musikforschung 2019 in Paderborn und Detmold, Bd. 1), Detmold 2020, S. IX-X, DOI: 10.25366/2020.43.

1 Freigestellt war den Autor*innen auch, ob sie sich für eine gendersensible Sprache entscheiden bzw. welche Form des Genderns sie bevorzugen. 